\title{
Index to Volume 22 of Oryx, 1988
}

Acacia saligna, 114

Acidification, 4

Acinonyx: jubatus, 113; jubatus venaticus, 115

Addax, 112

Addax nasomaculatus, 112

Agapornis: cana, 16; fischeri $\times$ personata, 142

Ailuropoda melanoleuca, 143, 232

Aldabra, threat to, 15-17

Alopex lagopus, 111

Alouatta seniculus, 11, 14

Alytes muletensis, 249-250

Ambystoma maculatum, 132

Ancistrocactus tobuschii, 149

Animal Smugglers, The (book review), 128-129

Ankarana, Madagascar, 163-171, 240-241

Anser albifrons flavirostris, 140

Antelope, giant sable, 142

Anthrax, in rhino horn, 231

Antilope cervicapra, 233

Antwren, black-hooded, 119

Aphelocoma c. coerulescens, 118

Aplothorax burchelli, 231

Aral Sea, USSR, 140

Arctic, damage to wildlife, 235

Ashby, K. R., The clouded leopard in Sumatra, 44-45

Ass, Asiatic wild, $85 \sim 88$

Assumption Island, 15-17

Astralagus robbinsii var. jesupi, 118

Ateles paniscus paniscus, 11,14

Atelocynus microtis, 96

Australia, move for endangered species, 59

Avahi laniger laniger, 165

Aye-aye, 166, 240

Baal, F. L. J., Mittermeier, R. A. and van Roosmalen. M. G. M., Primates and protected areas in Suriname, 7-14

Babbler, Steere's, 143

Balmford, A., A future for Palawan's forests?, $30-35$

Bat: Indiana, 148; Ozark big-eared, 149

Batisse, M., 187

Bats, killed by DDT, 146

Bear: brown in Poland, 141; grizzly, 235; polar, 146

Beetle, giant ground, 231

Belize, and Coca-Cola, 119

Bennett, E. L., Proboscis monkeys and their swamp forests in Sarawak, 69-74

Bern Convention, 230

Bertram, B., 60

Bird of Time: The Science and Politics of Nature Conservation. The (book review), 182
Birds to Watch: The ICBP World Check-List of Threatened Birds (book review), 245

Birgus latro, 53, 238

Blackbuck, 233

Boa, Round Island, 18, 19,21, 22, 180

Bone, Q., Sharks: A Photographer's Story (book review), 122

Bonin Islands, Japan, flora, 143

Bos sauveli, 145

Boycott: of Burger King, 56; ivory, 229; tuna, 236

British Columbia's wolf policy (letter), 48

Bromus mango, 151

Bruno H, Schubert Prize, 121

Bubulcus ibis, 16

Buceros hydrocorax, 101

Bucorvus leadbeateri, 113

Budowski, G., 186

Bufo hemiophrys, 147

Bulbul: Malagasy, 17; red-whiskered, 16,17

Bunolagus monticularis, $\overline{5} 1$

Burton, J. A., 246

Burton, J. A., Encyclopedia of Indian Natural History (book review), 126; Extinct Birds (book review), 128; The Mammals of Nigeria (book review), 129

Burton, R., In the Shadow of Fujisan, Japan and its Wildlife (book review), 63

Bustard: Arabian, 232; Houbara, 116

Butterfly: Eltham copper, 59; monarch, $56,57,235$

Cactus: San Rafael, 149; Tobusch fishhook, 149

Caiman, black, 96

Cairina scutulata, 144

Calidris pusilla, 146

Cambarus zophonastes, 56

Campephilus principalis, 58

Canis: lupus, 56, 229; lupus baileyi, 150; rufus, 150; simensis, 142

Cantharellus cibarius, $3-4$

Captive-breeding: 172-178; of blackfooted ferrets, 56 ; of brow-antlered deer, 233; of Californian condor, 149,235 ; of freckled duck, 59; of giant clams, 238; of invertebrates, 231; of maleo fowl, 55 ; of northern aplomado falcon, 236; of

Przewalski's horse, 153-157; of whales, 143

Capuchin: brown or tufted, 14 ; weeper, 13, 14

Caretta caretta, 230

Caribou: Peary, 146; Porcupine, 55

Casarea dussumieri, 18, 19, 180

Cat: sand, 115; wild, 111

Catagonus wagneri, 120
Cebus: apella apella, 11, 14 nigrivittatus, 11,14

Ceratogynus spp., 115

Ceratotherium simum, 51, 231

Cercocarpus traskiae, 236

Cercopithecus: diana, 51; solatus, 190

Cervus eldi eldi, 233

Chable, P., 187

Chanterelle, 3-4

Cheatum, E. L., 153

Cheetah: 113; Asiatic, 115; in captivity, 56

Cheirogalus major, 165

Chelonia mydas, 53

Chimpanzee, western, 51

Chiropotes satanus chiropotes, 11, 14

Chivers, D. Forest Life and Adventures in the Malay Archipelago (book review), 244-245; The Natural History of Domesticated Mammals (book review), 243-244

Chlamydotis undulata, 116

Choeropsis liberiensis, 50

Choriotis arabs, 232

Choudhury, A. Priority ratings for conservation of Indian primates, 89-94

Cirsium vinaceum, 118

CITES, see Convention on International Trade in Endangered Species of Wild Fauna and Flora

Civet, Malabar, 116

Clam, giant, 238

Clover, running buffalo, 118, 148

Cnemidophorus gramivagus, 151

Coleman, B., 130

Collins Guide to the Rare Mammals of the World (book review), 245

Colobus badius, 51

Colobus, red, 50, 51

Condor, Californian, 149, 235

Conservation in Africa; people, policies and practice (book review), 181-182

Conserving India's Natural Heritage (book review), 126

Contraception, in wild animals, 196

Conure, greater Patagonian, 58

Convention on the Conservation of European Wildlife and Natural Habitats (Bern Convention), 230

Convention on International Trade in Endangered Species of Wild Fauna and Flora, 110, 229, 232, 235

Convention for the Protection of the Natural Resources and Environment of the South Pacific Region, 120

Convention on Wetlands of International Importance especially as Waterfowl Habitat (Ramsar Convention), 110, 141, 211

Cooper, J. E. and West, C. D. Radiological studies on endangered Mascarene fauna, 18-24 
Corals, destruction in Thailand, 234

Corruption, of wildlife officials, 231

Corvus: alba, 16; hawaiiensis, 119

Corypha umbraculifera, 144

Cougar, 149

Coypu, 229

Crab, coconut or robber, 53,238

Crane: eastern sarus, 145; Mississippi sandhill, 56; whooping, 56, 57, 149

Crassula helmsii, 49

Crayfish, cave, 56

Crescentia portoricensis, 150

Crocodile: mugger, 233; Nile, 168; saltwater, 233

Crocodylus: palustris, 233; porosus, 233

Crow: Hawaiian, 119; pied, 16

Cull, of seals, 235

Curieuse Island, Seychelles, 4-5, 240

Curlew, eskimo, 118, 146

Curry-Lindahl, K., 186

Cyanoliseus patagonus byroni, 58

Cyathea dryopteroides, 118

Dace, blackside, 118

Dacrycarpus dacrydioides, 60

Dalyan beach, Turkey, 230

Dams: Besul, Malaysia, 145; Cliff, USA 119; Nam Choan, Thailand, 54, 144, 191-192; in Saskatchewan, Canada, 146

Danaus plexippus, $56,57,235$

Daniel, J. C., 187

Dasmann, R., 186

Daubentonia madagascariensis, 166 240

Debt-for-Nature Swaps, 58, 234, 239

Deer: Manipur brow-antlered, 233; musk, 232; Père David's, 53

Dendroica kirtlandii, 147

Dicerorhinus sumatrensis, 118,145 , 241

Diceros bicomis, 51, 142, 143, 231

Dictyosperma album var. conjugatum, 62

Dieback, forest, 3-4

Dihaila Jheel, India, 211-215

Dioxin, 55

Diseases of wildlife: canine parvovirus, 56; heartworm, 56; at Stillwater Wildlife Management Area, USA, 56; unknown in cranes, 56

Dog: African hunting, 51, 113; ban on hunting of African hunting dog, 121; bush, 96; small-eared, 96

Dolphin: bottle-nosed, 113; deaths in nets, 236

Dormouse, 111

Dove: barred ground, 15, 16; Malagasy turtle, 16

Dowsett-Lemaire, F. and Dowsett, R. J., Threats to the evergreen forests of southern Malawi, 158-162
Drought, US, 235

Duck: freckled, 59; white-winged wood, 144

Dugong, 115, 120

Dugong dugon, 120

Dupuy, A. R., 121

Eagle: harpy, 96; Madagascar fish, 231; Philippine, 103; white-tailed sea, 229

Earwig, giant, 142, 231

Ecology of Sumatra, The (book review), 182-183

Edwards, I., The Future of the Tropical Rain Forest, 192-193

Egg-collecting, 49

Egret, cattle, 16

Ehrlich, P. R., 121

Eidolon helvum dupraneum, 168

Elaphurus davidianus, 53

Elephants: African Elefund, 68; Knysna, 114

Elliott, H., 186

Elliott, S., Thai forest wins reprieve from dam, 191-192

Eltringham, S. K., Kangaroos: Their Ecology and Management in the Sheep Rangelands of Australia (book review), 62; Rangelands: A Resource Under Siege (book review), 124

Encyclopedia of Indian Natural History (book review), 126

Enhydra lutris nereis, 149

Environmental damage, at Prudhoe Bay, 235

Epioblasma penita, 55

Equus: przewalskii, 154; quagga, 114; zebra zebra, 143

Erymnochelys madagascariensis, 232

Eschrichtius robustus, 236

Estrilda astrild, 16

Exsul singularus, 120

Extinct Birds (book review), 128

Extinctions: 205-210; of earwig and beetle, 231

Falco: femoralis septentrionalis, 236; punctatus, 18, 19

Falcon, northern aplomado, 236

Fauna of Saudi Arabia (book review) 125

Felis: concolor, 149; concolor coryi 149; lynx, 112; margarita, 115; yagouaroundi, 96

Fern, Aleutian shield, 146

Ferrets, black-footed, 56, 148

FFPS: 232; Annual Report and Accounts, 1987, i-xui (between 162 and 163); Bat Project, 248; Council Members, 130-131; Executive Director, 188; Flora Project, 133; and fur trade in Kashmir, 135; Herpetofauna Programme, 248;
Inc., 132, 249; membership subscriptions, 246; Mountain Gorilla Project, 6, 135; Oryx 100\% Fund grants, 67-68, 132, 187-188, 246248; Toads on Roads campaign, 249; Wildlife Photographer of the Year Competition, 64-66

Fire: in Galàpagos, 237; in El Salvador, 236

Fish: cave, 168; conservation in South Africa, 114

Fitter, M., 185, 186, 187

Fitter, R., 184-185, 186, 187

Fleming, C., 121

Flowers, wild in US, 148

Fody: Malagasy, 16; red-headed forest, 17; Rodrigues, 18-22

Forest Life and Adventures in the Malay Archipelago (book review), 244-245

Forests: in Greece, 141; of southern Malawi, 158-162; swamp in Sarawak, 69-74

Foudia: eminentissima, 17; flavicans, 18, 19; madagascariensis, 16

Fox: Arctic, 111; red, 111

Fragments of Paradise (book review), $1-3$

Freira, 112

Friends of the Earth, 141

Frog, leopard, 57

Fruit bat, Rodrigues, 18, 19, 22, 23

Fynbos, 114

Gambusia: amistadiensis, 149; nobilis, 149

Gavialis gangeticus, 233

Gecko: Round Island, 18, 19, 180; Serpent Island, 180

Geocarpon minimum, 118

Geochelone: elephantopus, 151; gigantea, 4, 240

Geopelia striata, 15

Gharial, 231

Gibbon, hoolock, 89

Globicephala melaena, 111

Glossogobius spp., 168

Goby, a blind, from Madagascar, 171

Goeldlin, P., 186

Gonometa postica, 52

Good Wood Guide, The, 141

Gooders, J., 188

Gooders, J., From the Director's desk, 189; Save the Birds (book review), 61

Goose, Greenland white-fronted, 140

Gopherus polyphemus, 150

Gorilla gorilla beringei, 5

Gorilla, mountain, 5-6

Grant, Lt Col J. P. of Rothiemurchus, 67

Grebe, hooded, 152, 153

Green, M. J. B., Conserving India's Natural Heritage (book review), 126 Oryx Vol 22 No 4, October 1988 
Greenwood, J., 60

Grus: americana, 56, 57, 149; antigone sharpii, 145; canadensis, 56

Guenon, sun-tailed, 190

Guide to the Fishes of Lake Malawi

National Park (book review), 183

Guillemot, Iberian, 230

Gull, Audouin's, 112

Gymnogyps califomianus, 149, 235

Gypaetus barbatus, 112

Haliaeetus: albicilla, 229; vociferoides, 231

Halichoerus grypus, 235

Haliotis tuberculata, 111

Hapalemur: aureus, 137; griseus, 240; simus, 137, 165, 166

Harpactira spp., 115

Harpia harpyja, 96

Harrison, M. J. S., The mandrill in Gabon's rain forest; ecology, distribution and status, 218-228;

New guenon from Gabon, 190-191

Harrison, T., British Columbia and its wolves (letter), 179-180

Hess, J., Twin birth in wild mountain gorillas, 5-6

Hide, D., The New Environmental Age (book review), 242

Higuero de Sierra, 150

Hill-partridge, Sichuan, 216-217

Hippocamelus bisulcus, 151

Hippopotamus, pygmy, 50

Hippotragus niger variani, 142

Hochbaum, H. A., 153

Holdgate, M., 60

Holly, Cook's, 118

Holt, W. V. and Moore, H. D. M., Semen banking-is it now feasible for captive endangered species?, 172-178

Hornbill: rufous, 101; southem ground, 113

Hornsby, T. R., 153

Horse, Przewalski's, 154-157

Huemel, 151

Humphries, C., 130-131

Hunting, of snow leopard, 53; see also Killing

Hunting of the Whale: a tragedy that must end, The (book review), 242243

Hylobates hoolock, 89, 92, 93, 94

Hyophorbe amaricaulis, 52

Hypsipetes madagascariensis, 17

Ibis, oriental or Japanese crested, 53, 143

Ilex cookii, 118

In the Shadow of Fujisan, Japan and its Wildlife (book review), 63

India, primates in, 89-94

Indonesia, forest plan, 234

Index
International Association for Research on, and Conservation of,

Endangered Cichlids, 229

International Neotropical Bird Society, 140

International Tropical Timber

Organization, 110, 193

International Whaling Commission, 49, 110, 111, 140, 195-196

Introductions: Australian stonecrop, 49; birds to Assumption, 15-17; bitou bush, 120; Chrysanthemoides monilifera, 120; coypu, 229; hybrid lovebirds, 142; Port Jackson willow, 114; rabbits, Round Island, 52; rats, 238; red shiners, 57 ; sensitive mimosa, 120

International Union for Conservation of Nature and Natural Resources:

General Assembly, 185-187, 232;

Valour Award, 121

IUCN Red List of Threatened Animals, 1988 (book review), 245

Ivory, vegetable, 50

Jackal, Simien, 142

Jackman, B., Okavango, Jewel of the Kalahari (book review), 127

Jaguar, 96

Jaguarundi, 96

Jarvis, C., Plants in Danger: what do we know? (book review), 61-62

Jay, Florida scrub, 118

Jennings, M. C., Fauna of Saudi Arabia (book review), 125

Jersey Wildlife Preservation Trust, 18

John C. Phillips Memorial Award, 187

John Paul Getty Prize, 121, 153

Jones, C. G., Round Island boa eats Serpent Island gecko (letter), 180

Jones, D., 185

Kangaroos: Their Ecology and Management in the Sheep Rangelands of Australia (book review), 62

Kassas, M., 186

Kestrel, Mauritius, 18, 19, 21

Killing: animals by army, Sudan, 231; illegal, of animals, US, 147; of birds in Cyprus, 230; illegal, of birds in Turkey, 230; of bottlenosed dolphins, 113; of crocodiles in Queensland, 152; of Greenland white-fronted goose, 140; of animals in Saudi Arabia, 142; of polar bears, 146; of porpoises by tuna fleet, 147 ; of seals, 152, 235; wildfowl, public opinion of, 120 ; wolves, 146, 235

King, B. and Li Guiyan, China's most endangered galliform, 216-217

Kingdon, J., Conservation in Africa; people, policies and practice (book review), 181-182

Kouprey, 145

Labidura herculeana, 142, 231

Lake: Manzala, Egypt, 230; Naivasha, 142

Lampsilis orbiculata, 148

Langur: capped, 89; golden, 89; Hanuman, 89; Nilgiri, 89

Larus audouinii, 112

Lead-shot, ban, 49

Legislation: animal protection, Andorra, 230, Catalan, Spain, 230; banning killing cetaceans, Brazil, 119; Endangered Species Act, USA, 147; environmental, Mexico, 236; on forests, Greece, 141; hunting ban, Pakistan, 232; on logging, Thailand, 145; to protect panda, 143; to protect wild dog, 51; Wildlife and Countryside Act, 1981 (UK), 111

Leiolopisma: acrisanum, 238; telfairii, 18,19

Lemur: coronatus, 164, 240; fulvus sanfordi, 164, 240

Lemur: broad-nosed gentle, 165; crowned, $164,165,170,240,241$; eastem woolly, 165 ; greater bamboo, 137; greater dwarf, 165; grey gentle, 137, 240; grey mouse, 165; Sanford's, 164, 170, 240; sportive, 165

Leontopithecus rosalia, 238

Leopard: clouded, 44-47; snow, 117, 197-204, 232

Lepidomeda vittata, 149

Lepilemur septentrionalis ankaranensis, 165

Leucopsar rothschildi, 118

Lewis, R. E., Mt Apo and other national parks in the Philippines, 100-109

Lizard, whiptail, 151

Logging: arrests in Sarawak, 117; in Australia, 238; in Malaysia, 54; stopped in Nicaragua, 58; Rennell Island, 121 ; Thai villagers stop, 54 ; in Thailand, 234

Loosestrife, rough-leaved, 118

Loris: Ceylon mountain slender, 194; slender, 89; slow, 89

Loris: tardigradus, 89, 91, 92; tardigradus nyticeboides, 194

Loutit, B., 187

Lovebird, 16

Lovejoy, T., 239

Lowe-McConnell, R., A Guide to the Fishes of Lake Malawi National Park (book review), 183

Lucas, G., 121, 187

Lupinus aridorum, 55

Lycaon pictus, 51, 113, 121 
Lyle, R., British Columbia and its wolves (letter), 179

Lynx, 112

Lysimachia asperulaefolia, 118

Macaca: arctoides, 89-94; assamensis, 89-94; fascicularis, 89-93; mulatta, 89-94; nemestrina, 89-94; radiata, 89, 93; silenus, 89-94; sylvanus, 50

Macaque: Assamese, 89; Barbary, 50; bonnet, 89 ; lion-tailed, 89 ; longtailed or crab-eating, 89 ; pig-tailed, 89; stump-tailed, 89, 93

Macrocephalon maleo, 55

Mahogany, Catalina, 236

Mai Po Marshes, 53

Malawi, forests in, 158-162

Malaxis boninensis, 143-144

Maleo fowl, 54, 55

Mammals of Nigeria, The (book review), 129

Manatee, 75-84, 150

Mandrill, 218-228

Mandrillus sphinx, 218-228

Mangifera spp., 145

Mango, 145

Mangrove, in Sarawak, 69-74

Margaritifera margaritifera, 111

Mauritius, endangered fauna, 18-24

Megapode, Nicobar, 144

Megapodius freycinet, 144

Megaptera novaeangliae, 119

Melanosuchus niger, 96

Melastoma tetramerum, 143

Menidia extensa, 56

Mesite, white-breasted, 163, 167, 240

Mesitomis variegata, 167, 240

Microcebus murinus, 165

Migratory birds, conservation of, 140

Milk-vetch, Jesup's, 118

Miniopterus: minor manaui, 168; inflatus africanus, 168

Minnow; glass, 56; woundfin, 57

Mishra, H., 121, 153

Monachus monachus, 230

Monkey: bear, 194; Diana, 51; black snub-nosed, 41-43; black spider, 14; Phayre's leaf, 89; proboscis, 69-74; red howler, 14 ; rhesus, 89 ; squirrel, $10,12,13$

Monkey Mia, 120

Morris, P., The Animal Smugglers (book review), 128-129; The Natural History of Whales and Dolphins (book review), 122-123

Moschus moschiferus, 232

Moth, silk-producing, 52

Mulvaney, K., The Sei Whale: Population Biology, Ecology and Management (book review), 123124; Whalers treading water, 195 196
Mussel: Curtus's, 55; Judge Tait's, 55; Marshall's, 55; pearl, 111; penitent, 55 ; pink mucket pearly, 148

Mustela nigripes, 56,148

Myers, N., Mass extinction-profound problem, splendid opportunity, 205-210; Serengeti (book review), 127

Mynah, Rothschild's, 118

Myocaster coypus, 229

Myotis sodalis, 148

Myrmotherula erythronotos, 119

Nactus serpeninsula, 180

Nasalis larvatus, 69

National parks and nature reserves, see Protected areas

Natural History of Domesticated Mammals, The (book review), 243244

Natural History of Whales and Dolphins, The (book review), 122123

Nduku, W. L., 121

Nectarinia sauimanga abbotti, 16

Neofelis nebulosa: brachyurus, 46 ; diardi, 44

Nepal, wildlife in, 233

Nesoenas mayeri, 18, 19

New Environmental Age, The (book review), 242

New species: blind goby, 171; guenon, 190; lemur, 137; lizard, 151

Nicholls, F., 186

Nipponia nippon, 53, 143

Notropis: lutrensis, 57; mekistocholas, 149

Numenius borealis, 118,146

Nycticebus coucang, 89-94

Okavango, Jewel of the Kalahari (book review), 127

Oldfield, S., Plant Conservation in California (book review), 138-139

Olea europaea, 143

Olive, 143

Orchids, thefts of, 111

Oreodaimon quathlambae, 115

Ormer, Channel Islands, 111

Ormerod, W. E. and Rickman, L. R., Sleeping sickness control-how wildlife and man could benefit. $36-40$

Oryx: Arabian or white, 52; scimitarhorned, 112

Oryx: dammah, 112; leucoryx, 52

Otter: Californian or southern sea, 56 , 149; giant river, 96

Ovis canadensis, 118

Owl: barn, 141; northem spotted, 148

Palawan Island, Philippines, 30-35
Palm: bottle, 52; hurricane, 52 ; in

Malaysia, 117; talipot, 144

Pan troglodytes, 51

Panda, giant, 49, 143, 232

Panther, Florida, 149

Panthera: onca, 96; uncia, 198, 232

Paphiopedilum malipoense, 115

Paraguay, Nature Conservation Foundation, 237

Parakeet, Mauritius, 143

Paralucia pyrodiscus lucida, 59

Partula spp., 135-136

Parus holsti, 143

Pavo muticus, 234

Peafowl, green, 234

Peccary, Chacoan, 120

Pediocactus despainii, 149

Pelecanus erythrorhynchos, 55

Pelican, American white, 55

Peltophryne lemur, 150

Penguin, jackass, 113

Penstemon haydenii, 149

Pesticides: database, 110; DDT and bats, 146

Peter Scott Merit Award, 187

Petrogale xanthopus, 59

Petroica traversi, 120

Pheasant, Sichuan, 49

Phelsuma guentheri, 18, 19, 180

Philippines, forest in, 30-35; national parks in, 100-109

Phoca groenlandica, 235

Phocoena sinus, 236

Phoxinus cumberlandensis, 118

Phytelephas macrocarpa, 50

Picoides: borealis, 119; leucotus, 229

Piddington, K., 153

Pigeon, pink, 18, 21, 29

Pine marten, 111

Pithecia pithecia, 11, 13

Pithecophaga jefferyi, 102

Pitta gurneyi, 145

Plagopterus argentissimus, 57

Plant conservation: in California, 138139; in Japan, 116

Plants in Danger: what do we know? (book review), 61-62

Plecotus townsendii ingens, 149

Pleurobema: curtum, 55; marshalli, 55; taitianum, 55

Poaching: of cactus, 149 ; of rhino, 142 143; see also Killing

Podiceps gallardoi, 152

Pollution; Calancan Bay, Philippines, 118; at Stillwater Wildlife Management Area, US, 56; TBT, 57

Polystichum aleuticum, 146

Porpoise, Gulf of California, 236

Pop, I., 239

Potoroo, long-footed, 238

Potorous longipes, 238

Presbytis: entellus, 89-93; geei, 89-93; johnii, 89-94; phayrei, 
89-94; pileatus, 89-94; vetulus monticola, 194

Primates: in Gabon, 225; in India, 89-94; in Suriname, 7-14

Príncipe, birds on, 112

Propithecus: diadema, 165; diadema perrieri, 240

Protected areas: in Africa, 112; Air

Tenere, Niger, 142; Ankarana

Special Reserve, Madagascar, 163-171, 240-241; in Australia,

120; Bale Mountains National Park, Ethiopia, 142; Bigi Pan, Suriname,

151; Brier Island, Nova Scotia, 146;

Bruce Peninsula, Canada, 146;

Cahuinari National Park, Colombia,

119, 151; Chernobyl Ecological

Reserve, USSR, 229; Chinji National

Park, Pakistan, 144; Coed-y-cerrig

National Nature Reserve, UK, 141;

Coloangubra National Park,

Australia, 238: Daintree,

Queensland, 120; Dinder National

Park, Sudan, 112; Dja Faunal

Reserve, Cameroon, 110; Dudhwa

National Park, India, 117; Egan

Peaks National Park, Australia, 238;

Endau Rompin National Park,

Malaysia, 137-138; Filfla Nature

Reserve, Malta, 141; in Finland, 140

fire in El Salvador, 236; in Grenada,

151; Gros Morne National Park,

Canada, 110; Hawaii Volcanoes

National Park, 110; Hol Chan

Marine Reserve, Belize, 57; Horton

Plains National Park, Sri Lanka,

193-194; Kakadu National Park,

Australia, 110; Karera Bustard

Sanctuary, India, 211; Kaziranga,

India, 53; Kennedy Coulee, Alberta,

55; Kushiro Marshland, Japan, 116;

land swap in US, 148; Langebaan

Lagoon National Park, South Africa,

114; Little Karoo, South Africa, 52 ;

Lope Reserve, 191; Mai Po, 232;

Manu National Park, Peru, 110;

Mary's Point, Canada, 146; marine reserve, USSR, 229; Mombasa

Marine National Park, Kenya, 51; Mt

Apo, Philippines, 100-109; Mt

Kilimanjaro National Park, Tanzania,

110; Mt Taishan, China, 110; Murrah

Nature Reserve, Australia, 238;

North Cape Scientific Reserve, 60;

plan to privatize national nature

reserves (UK), 111; Peace Park

Nicaragua/Costa Rica, 150; Rudall

River National Park, Australia, 152;

Sanctuario Nacional de Ampay,

Peru, 237; Sanctuario Nacional Los

Manglares de Tumbes, Peru, 237;

sanctuary for dugongs, 120

Shannon Karri National Park,
Australia, 152; Shaumari Wildlife Reserve, Jordan, 52; Sian Ka'an Biosphere Reserve, Mexico, 110, 119; South Moresby National Park, Canada, 55; Sporades National

Marine Park, Greece, 230;

Sundarbans National Park, India, 110; in Suriname, 7-14; in Swedish Arctic, 140; Tallgrass Prairie National Preserve, US, 149; Tambopata Reserve Zone, Peru, 95-99; Tantawangalo National Park, Australia, 238; Tasman Wilderness, New Zealand, 59; Tatama National Park, Colombia, 51, 119; Tiwai Island, Sierra Leone, 50; Turkish wetlands, 230; Uluru National Park Australia, 110; Utria National Park, Colombia, 119, 151; Valia-Kalda National Park, Greece, 25-29; Vercors, France, 141; VictoriaRandenigala-Rantambe Sanctuary, Sri Lanka, 234; Whanganui National Park, New Zealand, 59; Wild Ass Sanctuary, India, 85, 86; Yanachaga Chemillen National Park, Peru, 58, 153; Yasuni National Park, Ecuador, 58; Yurnurtalik Lagoons, Turkey, 230.

Pseudemys alabamensis, 181

Psittacula eques, 143

Pterinochilus, 115

Pterodroma: madeira, 112; magentae, 120

Pteronura brasiliensis, 96

Pteropus rodricensis, 18, 19

Ptychocheilus lucius, 57

Pusa hispida saimensis, 229

Pycnonotus jocosus, 16

Quadrula stapes, 55

Quagga, 114

Quinnell, R., A Future for Palawan's forests? 30-35

Rabbit, riverine, 51

Rabinowitz, A., The clouded leopard in Taiwan, 46-47

Rahmani, A. R., Dihaila Jheel-a new candidate for the Ramsar Convention, 211-215

Ramsar Convention, see Convention on Wetlands of International Importance especially as Wildfowl Habitat

Rana yavapaiensis, 57

Rangelands: A Resource Under Siege (book review) 124

Rangifer: tarandus, 55; tarandus pearyi, 146

Reeves, R. R., Tuboku-Metzger, D. and Kapindi, R. A., Distribution and exploitation of manatees in Sierra
Leone, 75-84

Reintroduction: addax, 112; Barbary macaques, 50 ; black rhino, 231; Cape mountain zebra, 143; crocodiles, 233; elephants, 52 ; golden lion tamarin, 237-238; Melastoma tetramerum, 143; Mexican wolf, 150; Père David's deer, 53; red wolf, 150; Przewalski's horse, 153-157; scimitar-horned oryx, 112; southern sea otter, 56 149; Texas snowbells, 57; whitewinged wood duck, 144

Rennell Island, Solomons, 121

Rhinoceros: 50; black, 51, 142, 143 , 231; Javan, 241; Sumatran, 118, 145, 241; white, 51, 231

Rhinoceros sondaicus, 241

Rhinopithecus bieti. 41

Roberts, P., Introduced birds on Assumption Island - a threat to Aldabra, 15-17

Robin, Chatham Island, 120

Rock-wallaby, yellow-footed, 59

Rookmaaker, L. C., The Indo-Chinese rhinoceros (letter), 241

Round Island, 18-24, 52

Rudge, J., The Hunting of the Whale: a tragedy that must end (book review), 242-243

Ryder, O. A., Przewalski's horseputting the wild horse back in the wild, 154-157

Sadio, M., 121

Saguinus midas midas, 11, 12

Saki, bearded, 13, 14; white-faced, 13 , 14

Saimiri sciureus sciureus, 11,12

Salamander, tunnels for, 132-133, 249

Salmo salar, 49

Salmon: Atlantic, 49; Baltic, 140; Pacific, 146-147

Samour, J., Is the tide turning for Curieuse? (letter), 240

Sancez, R., 239

Sandalwood, 238

Sandpiper, semi-palmated, 146

Santalum austrocaledonicum, 238

Santiapillai, C., The clouded leopard in Sumatra, 44-45

Sao Tóme, birds on, 112

Sarawak, logging, 234

Save the Birds (book review), 61

Schaller, G. B., Li Hong, Talipu, Ren Junrang and Qiu Mingjiang, The snow leopard in Xinjiang, China, 197-204

Scott, R. F., 187

Seal: grey, 235; harp, 235;

Mediterranean monk, 230; Saimaa, 229 
Seed banks and conservation, 139

Sei Whale: Population Biology, Ecology and Management, The (book review), 123-124

Serengeti (book review), 127

Serin, Mozambique, 16

Serinus mozambicus, 16

Shad, 52

Sharks, A Photographer's Story (book review), 122

Sheep, bighorn, 118

Shell, stirrup, 55

Shiner: Cape Fear, 149; red, 57

Sierra Leone, manatees in, 75-84

Sifaka: diadem, 165; Perrier's diadem, 240

Simeulue, Sumatra, 117

Skink, Round Island, 18, 19, 20, 21

Sleeping sickness, 36-40

Smielowski, J. M. and Raval, P. P., The Indian wild ass - wild and captive populations, 85-88

Snail, St Helena blushing, 231

Snake, garter, 54, 55

Snowbell, Texas, 57

Society for Wildlife Art of the Nations, 229

Species Survival Commission, 186 , 232

Speothos venaticus, 96

Spheniscus demersus, 113

Spider, baboon, 115

Spinedace, Little Colorado, 149

Squawfish, Colorado, 57

Squirrel, Mount Graham red, 118

Sri Lanka, forest in, 234

Steel shot, 235

Steele, D., 131

Stemotherus depressus, 118

Stephanomeria malheurensis, 148

Stewart, P. D., Ankarana damaged (letter), 240-241; Tambopata

Reserve Zone, South-East Peru, 95-99

Stictonetta naevosa, 59

Stonecrop, Australian, in UK, 49

Streeter, D., The Bird of Time: The Science and Politics of Nature Conservation (book review), 182

Streptopelia picturata, 16

Strix occidentalis caurina, 148

Strong, M., 186

Studies of Mascarene Island Birds (book review), 62-63

Styrax texana, 57

Succinea spp., 231

Sunbird, Souimanga, 16

Suriname, primates and protected areas, $7-14$

Swaminathan, M. S., 187

Taiko, Chatham Island, 120
Takdjahn, A., 187

Talbot, L. M., 186

Tamarin: golden-handed, 10, 12; golden lion, 237

Tamiasciurus hudsonicus grahamensis, 118

Tanzanian Wildlife Society, 231

Tarsier, Philippine, 102

Tarsius syrichta, 102

Taxus sp. 119

Tello, J., 187

Thamnophis sirtalis, 55

Thistle, Sacramento Mountain, 118

Tigrisoma lineatum, 95

Tillandsia, 229

Tit, Formosan yellow, 143

Toad: Mallorcan midwife, 249-250; Puerto Rican crested, 150; tunnel, 249; Wyoming, 147

Tortoise: Aldabra giant, 4-5, 240; giant, 151; gopher, 150; village, 249

Tot, Kalang Anak, 60

Treaty of Protected Areas in the Mediterranean, 112

Tree-fern, elfin, 118

Triaenops persicus rufus, 168

Trichechus: manatus, 150; senegalensis, 75

Tridacna gigas, 238

Trifolium stoloniferum, 118, 148

Tropical Rain Forest, The Future of (conference report), 192-193

Tsetse control, 36-40, 113

Tsunis, G. L., The Valia-Kalda National Park, Greece, 25-29

Turner, I., The Ecology of Sumatra (book review), 182-183

Tursiops aduncus, 113

Turtle: Alabama red-bellied, 118; flattened musk, 118; freshwater Madagascar, 232; green, 53 ; loggerhead, 230

UK Dependent Territories, conservation in, 1-3

Uria aalge ibericus, 230

Ursus: arctos, 141,235; maritimus, 146

Varona, L., 239

Viverra civettina, 116

Vulpes vulpes, 111

Vulture, bearded, 112

Walker, J. H. C., British Columbia's wolf policy (letter), 48

Walker, T. A. P., 184

Warbler, Kirtland, 147

Warea amplexifolia, 55

Watts, D. P., Twin birth in wild mountain gorillas, $5-6$
Waxbill, common, 16

Wetlands: Camalti Tuzlasi, Turkey, 112; saved in Everglades, 150; Lake Manzala, Egypt, 230; Lake Shinji, Japan, 232; Long Pond, Pennsylvania, 147; Mai Po Marshes, 232; saved in Massachusetts, 147; in Turkey, 230; in UK, 140-141; in US, 147

Whale: grey, 236; humpback, 119 ; pilot, 111

Whaling: 195-196; ban on, 111; and Iceland, 49, 110; Japanese, 49, 110, 140

Whitaker, R. and Z., 187

Whitlam, G., 187

Wildlife trade: baboon spiders, 115; ban in Papua New Guinea, 121; bird exports from China, 53; birds of prey, Pakistan, 144; birds from Taiwan, 143; bulbs, Turkey, 133-134; butterflies, 116; in China, 115 ; coconut or robber crabs, 53 ; eagles, US, 119; garter snakes, 54 55; in Guyana, 237; ivory ban, 231; in Laos, 117; in musk, 232; orchids, 111; palms, 111 ; pandas, 115 ; in Philippines, 33; in rhino, 234; in reptiles, 235; sandalwood, 238; stuffed birds, Syria, 232; tiger skins, Singapore, 234; Tillandsia, 229; wallaby and kangaroo products, 49

Willow, Port Jackson, 114

Wilson, J., Sri Lanka declares a new national park, 193-194

Wilson, J. M., Stewart, P. D. and Fowler, S. V., Ankarana-a rediscovered nature reserve in northern Madagascar, 163-171

Wilson, R., Birds to Watch: The ICBP World Check-List of Threatened Birds (book review), 245; Studies of Mascarene Island Birds (book review), 62-63

Wire-lettuce, Malheur, 148

Wolf: 229; in British Columbia, 48, 179-180, 235; grey in Minnesota, 56; Mexican, 150; red, 150

Woodpecker: ivory-billed, 58; redcockaded, 119; white-backed, 229

World Bank, 58, 234

World Heritage Convention, 110, 229

World Wildlife Fund Gold Medal, 121

Yang De-hua, Black snub-nosed monkeys in China, 41-43

Yew, Pacific, 119

Yuhina brunneiceps, 143

Zambia, conservation in, 51

Zebra, Cape mountain, 143

Oryx Vol 22 No 4, October 1988 